\title{
SHIP TRAJECTORY TRACKING CONTROL SYSTEM DESIGN BASED ON SLIDING MODE CONTROL ALGORITHM
}

\author{
Yong Liu \\ Renxiang Bu \\ Xiaori Gao \\ Dalian Maritime University, China
}

\begin{abstract}
The paper reports the design and tests of the planar autopilot navigation system in the three-degree-of-freedom (3-DOF) plane (surge, sway and yaw) for a ship. The aim of the tests was to check the improved maneuverability of the ship in open waters using the improved nonlinear control algorithm, developed based on the sliding mode control theory for the ship-trajectory tracking problem of under-actuated ships with static constraints, actuator saturation, and parametric uncertainties. With the integration of the simple increment feedback control law, the dynamic control strategy was developed to fulfill the under-actuated tracking and stabilization objectives. In addition, the LOS (line of sight) guidance system was applied to control the motion path, whereas the sliding mode controller was used to emulate the rudder angle and propeller rotational speed control. Firstly, simulation tests were performed to verify the validity of the basic model and the tracking control algorithm. Subsequently, full scale maneuverability tests were done with a novel container ship, equipped with trajectory tracking control and sliding mode controller algorithm, to check the dynamic stability performance of the ship. The results of the theoretical and numerical simulation on a training ship verify the invariability and excellent robustness of the proposed controller, which: effectively eliminates system chattering, solves the problem of lateral drift of the ship, and maintains the following of the trajectory while simultaneously achieving global stability and robustness.
\end{abstract}

Keywords: Keywords: LOS; ship trajectory; tracking control system; sliding mode control

\section{INTRODUCTION}

Unmanned surface vessels (USVs) are attracting a great deal of attention from researchers all over the world because of their extensive applications in military reconnaissance, homeland security, shallow water surveys, environmental monitoring, and operational coordination with autonomous underwater vehicles (AUVs) [1-3]. Three types of control technologies: set-point control, trajectory tracking control, and path following control, play a crucial role in allowing USVs to achieve their specified tasks automatically [4]. Setpoint control [5-7] is important for dynamic positioning of a vessel in fixed target operations, such as autonomous docking. Trajectory tracking control [8-12] enables the ship to track the desired time-referenced trajectory. In the pathfollowing control scenario [13-14], a USV is required to follow the path at a certain speed without specified temporal constraints. The path is described by curve parameters, which are usually not time relevant. There are neither lateral nor vertical thrusters on most AUVs, and only longitudinal, yaw and pitch angle speeds are controlled directly. Therefore, the AUV is a typical under-actuated system, which makes trajectory tracking more difficult [15].

Trajectory tracking control of ships is especially difficult due to the effects of underactuativity [16-17] and nonholonomic constraint characteristics [18] of common surface ships, with the addition of the impact of nonlinearity[19], uncertainty[20], 
as well as wind and current susceptibility[21-23]. Normally these ships are not equipped with side thrusters, which might only be used when approaching or departing port. Reference [24] integrated the output redefinition method and the sliding-mode control technology, and applied them to the nonlinear non-minimum phase system design to acquire an asymptotically stable effect of ship straight-path tracking control. References [25] and [26] put forward a control algorithm taking into account current interference on the fourth order ship model, which estimates the uncertainty of the current interference. Reference [27] designed an output feedback controller on the basis of the nonlinear observer, and conducted the prototype model simulation experiment. The authors managed to perform straight-path tracking control globally and steadily in the case of external disturbances of wind, wave and current. Reference [28] integrated the Nussbaum technique into the backstepping method, and put forward a robust-adaptive fuzzy control algorithm, which enabled the ship cross track error to reach asymptotically a small neighborhood of the origin at exponential rate. To achieve trajectory tracking or positioning, various control strategies have been proposed, including robust adaptive control [29], sliding mode control [30-32], decoupling control[33], and adaptive neural control[34-37].

The maneuvering motion model proposed by the Japanese Ship Maneuvering Mathematical Model Group and called the MMG model is frequently used to simulate the motion of a ship. The MMG model divides the ship into ship hull, propeller, and rudder, and examines their performance. It can consider different values of fluid power that acts effectively on the ship. The MMG Group further improved the MMG model [38] by incorporating a method to correct the hydrodynamic derivatives in shallow water. As for ship motion guidance, LOS (line of sight) guidance is adopted, which can control the course between consecutive waypoints through correcting the cross-track error (XTE), and can also be used for tracking a moving target on the sea.

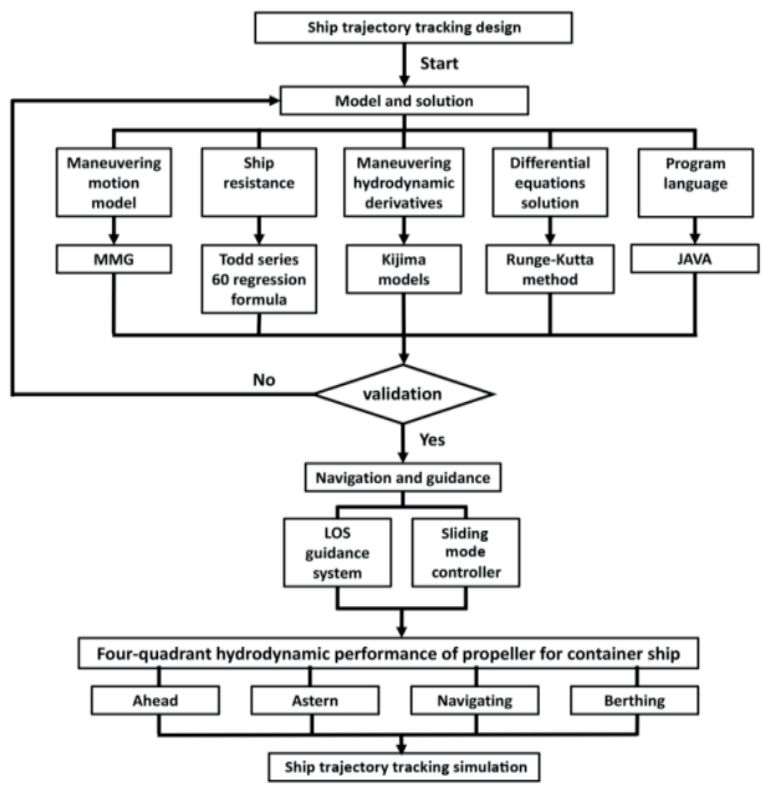

Fig. 1. Diagram of trajectory tracking control system design
The ship trajectory tracking control system is a closed loop feedback system, the aim of which is to reduce the tracking error. Once the tracking error meets the accuracy requirements, the program executes the next step until its completion. The trajectory tracking control system presented in the paper has been established for a container ship. The construction idea of this system is shown in Figure 1.

\section{MATHEMATICAL MODELING OF SHIP TRAJECTORY TRACKING}

\section{FIXED COORDINATE SYSTEM AND KINETIC COORDINATE SYSTEM}

Diagrammatic drawing of ship plane coordinates is shown in Figure 2. In the diagram, $x_{E} o_{E} y_{E}$ is the fixed coordinate system in which the axis $o_{E} x_{E}$ points to true north, and the axis $o_{E} y_{E}$ points to due east. $G$ is the ship's center of gravity, and its position is represented by $(x, y) ; \varphi$ is the course angle, and clockwise is positive; $V$ is the speed in tangent direction of ship's center of gravity over the ground in the plane, and $V x, V y$ are the projections in the body-fitted coordinate system. $U$ is the speed through water, and $\beta$ is the drift, defined as the intersection angle between the tangential direction of speed through water and the direction of ship fore and aft centerline, and port side is positive. $\varphi_{c}$ and $u_{c}$ represent the angle and rate of homogeneous flow, respectively.

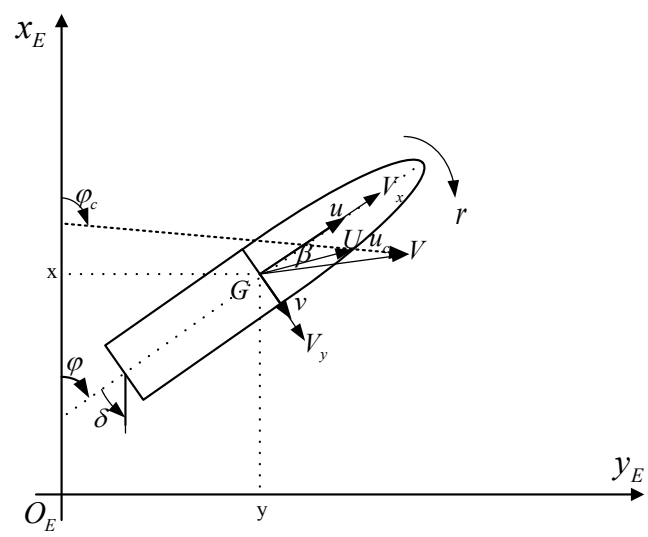

Fig. 2. Diagram of coordinate system

\section{DEFINITION OF SHIP TRAJECTORY TRACKING ERROR}

By simple coordinate transformation while maintaining generality, the ship lateral tracking error $y$ can be defined as the distance on the rhumb line from the ship to the line connecting two consecutive waypoints, as shown in Figure 3. 


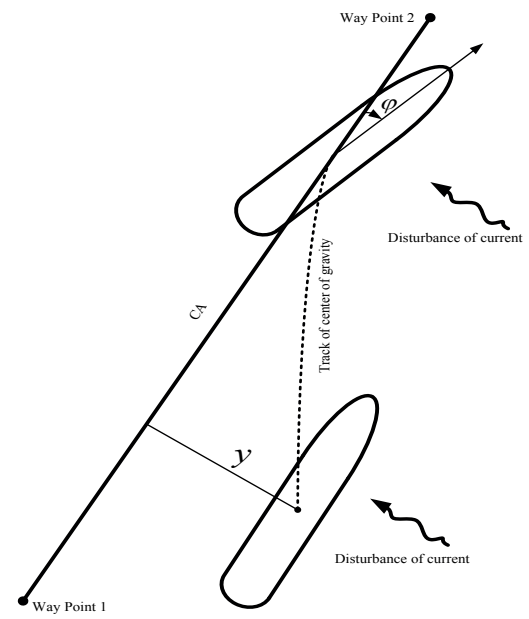

Fig. 3. Diagram of trajectory tracking control

\section{MATHEMATICAL MODEL OF SHIP TRAJECTORY TRACKING CONTROL}

When the ship sails at fixed speed at sea without controlling the longitudinal motion, the mathematical model of ship trajectory tracking control with the interference of wind and current can be expressed as:

$$
\left\{\begin{array}{l}
\dot{y}=u \sin \varphi+c(t) \\
\dot{\varphi}=r \\
\dot{r}=f(u, v, r)+g(u, v, r, \delta)+d(t) \\
\dot{\delta}=K_{E}\left(\delta_{R}-\delta\right) / T_{E} \\
c(t)=v \cos \varphi+u_{c} \sin \varphi_{c}
\end{array}\right.
$$

In this formula, $y$ is the transverse cross-track error, $u$ is the ship's speed through water, $v$ is the transverse speed through water, $r$ is the heading rate, $\delta$ is the rudder angle, $\delta_{R}$ is the commanded rudder angle, $T_{E}$ is the time constant of the steering gear, $f(u, v, r)$ and $g(u, v, r, \sigma)$ are the nonlinear functions containing $r$ and $\delta, d(t)$ is the unknown external disturbance torque, and $c(t)$ is the drift caused by the unknown current disturbance and ship transverse motion.

\section{SLIDING MODE CONTROLLER DESIGN AND STABILITY ANALYSIS}

\section{CONTROL OBJECTIVE AND ASSUMPTION TERMS}

The objectives of ship trajectory tracking control are: taking advantage of appropriate methods to determine $\delta$, and making the cross-track error $y$ approach zero. In other words, its goal is to make the ship sail on the scheduled track line. In the case of transverse wind and current disturbances, the ship needs to sail at a certain leeway and drift angle. In this case, the yaw rate approaches zero, while the course deviation $\varphi$ should not be zero.

According to practical conditions of navigation, let us make the following assumptions for system (1):

Assumption 1: $u \gg|u|, u \gg|v|$, which means that the longitudinal speed is far higher than the flow rate and the transverse speed;

Assumption 2: External interference is bounded;

Assumption 3: Ship course is controllable;

Assumption 4: $u, v, u_{c} r$ is bounded, and $u_{\text {max }}, v_{\text {max }}, u_{c \max }$, $r_{\max }$ are known;

Assumption 5: Lipschitz constants $L_{1}, L_{2}, L_{3}, L_{4}$ exist and satisfy the following inequation:

$$
\left\{\begin{array}{l}
\left|u_{t 2}-u_{t 1}\right| \leq L_{1} \Delta t \\
\left|c_{t 2}-c_{t 1}\right| \leq L_{2} \Delta t \\
\left|f\left(t_{2}\right)-f\left(t_{1}\right)\right| \leq L_{3} \Delta t \\
\left|d_{t 2}-d_{t 1}\right| \leq L_{4} \Delta t
\end{array}\right.
$$

Assumption 1 is easy to satisfy in normal weather and sea state, but when the transverse speed is higher than the longitudinal speed, the ship cannot keep the track. Assumptions 2 and 3 are the prerequisites for a controllable closed-loop system. Assumptions 4 and 5 are the inevitable results of action of the bounded ship control energy and the external interference energy.

\section{REDEFINITION OF OUTPUT VARIABLES AND ANALYSIS OF TRAJECTORY TRACKING ERROR STABILITY}

Considering practical conditions of wind and current disturbances, let us we redefine the output variables:

$$
Z=\varphi+k_{1} \arctan \left(k_{0} y\right)+k_{4} t h \int_{0}^{t}\left[\dot{y}+k_{3} t h\left(k_{2} y\right)\right] d_{t}
$$

where $\operatorname{th}(\cdot)$ denotes the hyperbolic tangent and $k_{0}, k_{1}, k_{2}$, $k_{3}, k_{4} \in \mathrm{R}^{+}$are the design parameters. The main reasons for adopting (3) are: 1) to control the ship track error by finding the nonlinear relationship between the heading $\varphi$ and the track error $y, 2)$ to ensure that the system is in a controllable state by using arc tangent and hyperbolic tangent functions with strict bounds in the range $(-1,1), 3)$ to ensure that the heading $\varphi$ and the track error $y$ are at the same balance point and to ensure the stability of the system, 4) to control the system convergence rate with $k_{1}, k_{3}, k_{4}$ and to adjust the slope of the curve with $k_{0}, k_{2}$.

When $\mathrm{Z}$ approaches zero:

$$
\varphi=-k_{1} \arctan \left(k_{0} y\right)-k_{4} t h \int_{0}^{t}\left[\dot{y}+k_{3} \operatorname{th}\left(k_{2} y\right)\right] d_{t}
$$


Making $\sigma=u \sin \varphi$, according to equations (1) and (4), we get:

$$
\left\{\begin{array}{l}
\dot{y}=\sigma+c(t) \\
\dot{\sigma}=-C \sigma-D k_{3} t h\left(k_{2} y\right)-C \cdot c(t)
\end{array}\right.
$$

In order to make the following formulas more clear, we use $C$ and $D$ to represent the complex variables in formula (5):
The above equation states that the ship has the heading angle $\varphi \neq 0$ in the presence of wind and current disturbances. Consequently, the ship needs to have a leeway angle while navigating at sea.

As such: $\dot{V}=-D \sigma k_{3} t h\left(k_{2} y\right)-C D y k_{3} t h\left(k_{2} y\right)<0$ always stands up.

In conclusion, when the global convergence of $Z$ approaches zero, $V=\frac{1}{2}(\sigma+C y)^{2}>0$ and $\dot{V}<0$, and the system (5) is asymptotically stable. For formula (4), in the presence of wind and current disturbances, the cross-track $C=\frac{u \cos \varphi \cdot k_{0} k_{1}}{1+\left(k_{0} y\right)^{2}}+\frac{u \cos \varphi \cdot k_{3}}{\operatorname{ch}^{2}\left(\int\left(\dot{y}+k_{3} t h\left(k_{2} y\right)\right) d_{t}\right)}, D=\frac{u \cos \varphi \cdot k_{3}}{\operatorname{ch}^{2}\left(\int\left(\dot{y}+k_{3} t h\left(k_{2} y\right)\right) d_{t}\right)}$ error $y$ is asymptotically stable and converging, and the track control is converted into the stabilization control of the intermediate variable $Z$.
In practical navigation, the leeway and drift angle is smaller than $90^{\circ}$, that is $\varphi \in(-\pi / 2, \pi / 2)$, then $\cos \varphi>0$ and, consequently, $C>0, D>0$.

Applying the Lyapunov function: $V=\frac{1}{2}(\sigma+C y)^{2}$ we get:

$$
\begin{aligned}
\dot{V} & =(\sigma+C y)(\dot{\sigma}+C \dot{y}) \\
& =\sigma \dot{\sigma}+C \sigma \dot{y}+C y \dot{\sigma}+C^{2} y \dot{y} \\
& =\sigma\left(-C \sigma-D k_{3} t h\left(k_{2} y\right)-C \cdot c(t)\right)+C \sigma(\sigma+c(t)) \\
& +C y\left(-C \sigma-D k_{3} t h\left(k_{2} y\right)-C \cdot c(t)\right) \\
& +C^{2} y(\sigma+c(t)) \\
& =-C \sigma^{2}-D \sigma k_{3} t h\left(k_{2} y\right)-C \sigma \cdot c(t) \\
& +C \sigma^{2}+C \sigma \cdot c(t)-C^{2} \sigma y-C D y k_{3} t h\left(k_{2} y\right) \\
& -C^{2} y \cdot c(t)+C^{2} \sigma y+C^{2} y \cdot c(t) \\
& =-D \sigma k_{3} t h\left(k_{2} y\right)-C D y k_{3} t h\left(k_{2} y\right)
\end{aligned}
$$$$
\because C>0, D>0 \text { and } \begin{cases}y>0, & k_{3} \operatorname{th}\left(k_{2} y\right)>0, \therefore-C D y k_{3} \operatorname{th}\left(k_{2} y\right)<0 \\ y<0, & k_{3} \operatorname{th}\left(k_{2} y\right)<0, \therefore-C D y k_{3} \operatorname{th}\left(k_{2} y\right)<0\end{cases}
$$

According to the order of magnitude: $\left|D \sigma k_{3} t h\left(k_{2} y\right)\right|<\left|C D y k_{3} t h\left(k_{2} y\right)\right|$. The proof is as follows:

$$
\begin{aligned}
& \left|D \sigma k_{3} \operatorname{th}\left(k_{2} y\right)\right|<\left|C D y k_{3} \operatorname{th}\left(k_{2} y\right)\right| \\
& \Leftarrow|\sigma|<|C y| \\
& \Leftarrow|u \sin (\varphi)|<\left|\left(\frac{u \cos \varphi \cdot k_{0} k_{1}}{1+\left(k_{0} y\right)^{2}}+\frac{u \cos \varphi \cdot k_{3}}{c^{2}\left(\int\left(\dot{y}+k_{3} t h\left(k_{2} y\right)\right) d_{t}\right)}\right) y\right| \\
& \Leftarrow|\sin (\varphi)|<\left|\left(\frac{\cos \varphi \cdot k_{0} k_{1}}{1+\left(k_{0} y\right)^{2}}+\frac{\cos \varphi \cdot k_{3}}{c h^{2}\left(\int\left(\dot{y}+k_{3} t h\left(k_{2} y\right)\right) d_{t}\right)}\right) y\right| \\
& \Leftarrow|\sin (\varphi)|<\left|\frac{\cos \varphi \cdot k_{0} k_{1}}{1+\left(k_{0} y\right)^{2}} y\right|+\left|\frac{\cos \varphi \cdot k_{3}}{c^{2}\left(\int\left(\dot{y}+k_{3} t h\left(k_{2} y\right)\right) d_{t}\right)} y\right| \\
& \Leftarrow|\sin (\varphi)|<|y|+1
\end{aligned}
$$

Formula (8) always holds.

Furthermore, If $y=0$, then $\dot{y}=\sigma+c(t)=0$ and $\sigma=-c(t)$. We arrive at: $u \sin \varphi=-c(t) \neq 0$.

\section{DESIGN OF SHIP TRAJECTORY TRACKING CONTROLLER BASED ON SLIDING-MODE METHOD}

For formula (3), in order to reduce the calculative complexity level in the process of working out $\delta$ as well as for the sake of simulation convenience, the control design is given in the following form:

$$
\left\{\begin{array}{l}
f_{1}(y)=k_{3} \operatorname{th}\left(k_{2} y\right)+\dot{y} \\
f_{2}\left(f_{1}, \varphi\right)=\varphi+k_{1} \arctan \left(k_{0} y\right)+k_{4} t h \int f_{1}(y) d_{t} \\
f_{3}\left(f_{2}\right)=k_{5} t h\left(f_{2}\right)+\dot{f}=k_{5} t h\left(f_{2}\right)+r+k_{1} \arctan ^{\prime}\left(k_{0} y\right)+k_{4} t h^{\prime} \int f_{1}(y) d_{t} \\
f_{4}\left(f_{3}\right)=k_{6} t h\left(f_{3}\right)+\dot{f}_{3}
\end{array}\right.
$$

Now, the control objective of the system is converted into the control of $f_{4}$, that is: when $f_{4}=0$, we get $f_{3}=0$ and $f_{2}=0$, that is:

$$
\varphi^{\rightarrow}-k_{1} \arctan \left(k_{0} y\right)-k_{4} t h \int_{0}^{t}\left[\dot{y}+k_{3} t h\left(k_{2} y\right)\right] d_{t}
$$

In the case of $f_{2}=0$, we have proved that the system is stable. After expanding $f_{4}\left(f_{3}\right)$ we get:

$$
\begin{aligned}
f_{4}\left(f_{3}\right) & =k_{6} \operatorname{th}\left(f_{3}\right)+\dot{f}_{3} \\
& =k_{6} \operatorname{th}\left(f_{3}\right)+k_{5} t h^{\prime}\left(f_{2}\right)+\ddot{f}_{2} \\
& =k_{6} \operatorname{th}\left(f_{3}\right)+k_{5} t^{\prime}\left(f_{2}\right)+\dot{r}+k_{1} \arctan ^{\prime \prime}\left(k_{0} y\right)+k_{4} t h^{\prime \prime} \int f_{1} d_{t} \\
& =k_{6} \operatorname{th}\left(f_{3}\right)+k_{5} t^{\prime}\left(f_{2}\right)+f(u, v, r)+f(u, v, r, \delta)+d(t) \\
& +k_{1} \arctan ^{\prime \prime}\left(k_{0} y\right)+k_{4} t h^{\prime \prime} \int f_{1} d_{t}
\end{aligned}
$$

Making $f_{4}\left(f_{3}\right)=0$, we get the control law in the presence of wind and current disturbances:

$\delta=f^{-1}(u, v, r, \delta)(f(\varphi(t), y(t))+f(u, v, r)+d(t))$

In this formula, $f^{-1}(u, v, r)$ is the indeterminate and complicated smooth and continuous function; $f(\varphi(t), y(t))$ is the 
nonlinear function with unknown structure which includes $\varphi$ and $y ; f(u, v, r)$ is the nonlinear function that includes $r$; and $d(t)$ is the unknown function.

Problems which appear in system (1) refer to parameter uncertainty as well as to wind and current disturbances. Moreover, the process of finding $\delta$ in formula (11) is rather complicated, which may lead to "calculation inflation". These disadvantages are not conducive to the realization of the simulation and actual application. To address the main goal of the path-following problem which is finding the nonlinear relationship between the rudder angle $\sigma$ and the track deviation $y$, we can use the intermediate variable $\dot{\sigma}$ to carry out the transition and ultimately determine this relationship. This paper adopts the following increment feedback control law [38]:

$$
\dot{\delta}=-p \cdot f_{4}-\varepsilon \operatorname{sgn}\left(f_{4}\right) \quad p, \varepsilon \in R^{+}
$$

In this formula, $p$ is the proportional feedback coefficient with a constant value.

\section{STABILITY ANALYSIS}

Substituting $\dot{y}=u \sin (\varphi)+c(t)$ into $f_{1}(y)=k_{3} t h\left(k_{2} y\right)+\dot{y}$, we get:

$$
f_{1}(y)=k_{3} t h\left(k_{2} y\right)+u \sin \varphi+c(t)
$$

Let us assume that for all $t \in\left[t_{1}, t_{2}\right], t_{2}=t_{1}+\Delta t$, if $f_{4}\left(t_{1}\right)>0$, $f_{4}\left(t_{2}\right)<0$, then, in line with function continuity, we can infer that there is a $\xi$ which $0 \leq \xi \leq 1$, when $t_{\xi}=t_{1}+\xi \cdot \Delta t, f_{4}\left(t_{\xi}\right)=0$, and for all $t \in\left[t_{1}, t_{\xi}\right], f_{4}(t)>0$. Then, when $t=t_{2}$, from formula (9) and the definition of derivative, we get:

$$
\begin{aligned}
\Delta f_{4} & =k_{6}\left[t h\left(f_{3}\left(t_{2}\right)\right)-\operatorname{th}\left(f_{3}\left(t_{1}\right)\right)\right]+k_{5}\left[t^{\prime}\left(f_{2}\left(t_{2}\right)\right)-t^{\prime}\left(f_{2}\left(t_{1}\right)\right)\right] \\
& +f\left(u, v, r\left(t_{2}\right)\right)-f\left(u, v, r\left(t_{1}\right)\right)+f^{-1}\left(u, v, r, \delta\left(t_{2}\right)-u, v, r, \delta\left(t_{1}\right)\right) \\
& +d\left(t_{2}\right)-d\left(t_{1}\right)+k_{1}\left[\arctan \left(k_{0} y\left(t_{2}\right)\right)-\arctan ^{\prime \prime}\left(k_{0} y\left(t_{1}\right)\right)\right] \\
& +k_{4}\left[t^{\prime \prime} \int f_{1}\left(t_{2}\right) d_{t}-t h^{\prime \prime} \int f_{1}\left(t_{1}\right) d_{t}\right]
\end{aligned}
$$

From formula (13), we get:

$$
\Delta \delta=\dot{\delta}\left(t_{\xi}\right) \Delta t=-\left[p \cdot f_{4}\left(t_{\xi}\right)+\varepsilon\right] \Delta t, 0 \leq \xi \leq 1,0<f_{4}\left(t_{\xi}\right)
$$

Substituting formula (16) into formula (15), and considering formula (2), we get:

$$
\begin{aligned}
\Delta f_{4} \leq & 2 k_{6}+2 k_{5}+L_{3} \Delta T-f^{-1} \cdot\left[p \cdot f_{4}\left(t_{\xi}\right)+\varepsilon\right] \Delta t+L_{4} \Delta t \\
& +2 k_{1} k_{0}\left[L_{1}+\left(k_{1} k_{0}+k_{4}\right) u_{c \max }+\left(k_{1} k_{0}+k_{4}\right) L_{2} \Delta t\right] \\
& +2 k_{4} k_{3} k_{2}\left(u_{\max }+u_{c \max }+v_{\max }\right)+2 k_{4}\left(L_{1}+k_{2} u_{c \max }+L_{2}\right)
\end{aligned}
$$

From the known conditions of Assumptions 1, 2, and 5, the leeway and drift angle does not exceed $90^{\circ}$ during the voyage, as well as $f_{4}(t)>0$. Consequently, when $t \in\left[t_{1} t_{k}\right]$, there is $p \in R^{+}$, render $\Delta f_{4}<0$; and when $t \in\left[t_{\xi} t_{2}\right]$, there is $f_{4}(t)<0$ and $p \in R^{+}$, render $\Delta f_{4}>0$.

In conclusion, $f_{4}$ is successive in the closed interval $\left[t_{1} t_{2}\right]$, and $f_{4}\left(t_{1}\right)$ and $f_{4}\left(t_{2}\right)$ are of opposite sign, then in the open interval $\left(t_{1}, t_{2}\right)$, there is at least one $\xi$ which renders $f_{4}(\xi)=0, f_{4}(t)$ and $f_{4}^{\prime}(t)$ are of opposite sign, then $f_{4}(t)$ is stable and converges to zero. Q.E.D.

\section{SHIP TRAJECTORY TRACKING CONTROL DESIGN SYSTEM}

\section{WAYPOINT LOS GUIDANCE}

LOS is used to control the course between two waypoints, thus making the ship navigate towards the next waypoint along the set route. After setting the expected course, LOS defines it as a function of the cross-track error, then calculates the error and corrects it by steering. Finally, navigation is achieved by making the variable heading value convergent. See Figure 4 for detail.

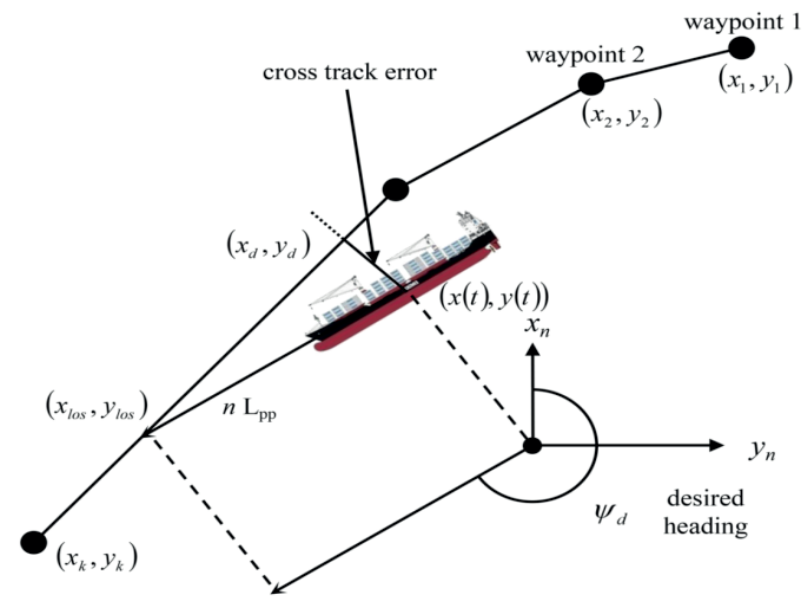

Fig. 4. Schematic diagram for LOS (line of sight)

In Figure 4, the ship position, usually measured with a satellite system, is $(x(t), y(t))$, while the next waypoint is $\left(x_{l o s}, y_{l o s}\right)$. Then the desired heading can be calculated as:

$$
\psi_{d}(t)=a \tan 2\left(y_{\text {los }}-y(t), x_{\text {los }}-x(t)\right)
$$

When the distance between the ship and the next waypoint is $n$ times larger than the ship length $L_{p p}$, then the LOS coordinate $\left(x_{\text {los }}, y_{\text {los }}\right)$ is given by:

$$
\left(y_{\text {los }}-y(t)\right)^{2}+\left(x_{\text {los }}-x(t)\right)^{2}=(n L p p)^{2}
$$


It is used to judge whether the target point has been reached and the guidance for next waypoint can start. Therefore, the smaller the value $n$, the higher the accuracy of guidance, but the difficulty increases if $n$ is too small, so we set $n=1$ in this paper.

\section{SHIP'S ROUTE PLANNING}

A simple route analyzed in this paper is shown in Figure 5. A coordinate system has been established with the initial position of the ship as the origin $(0,0)$. The set waypoints shown in the figure have been selected assuming that there are two islands on both sides of the ship route into port, and a narrow channel between them. Starting from the initial position, the ship is expected to go through waypoints 1,2 , 3 and 4 .

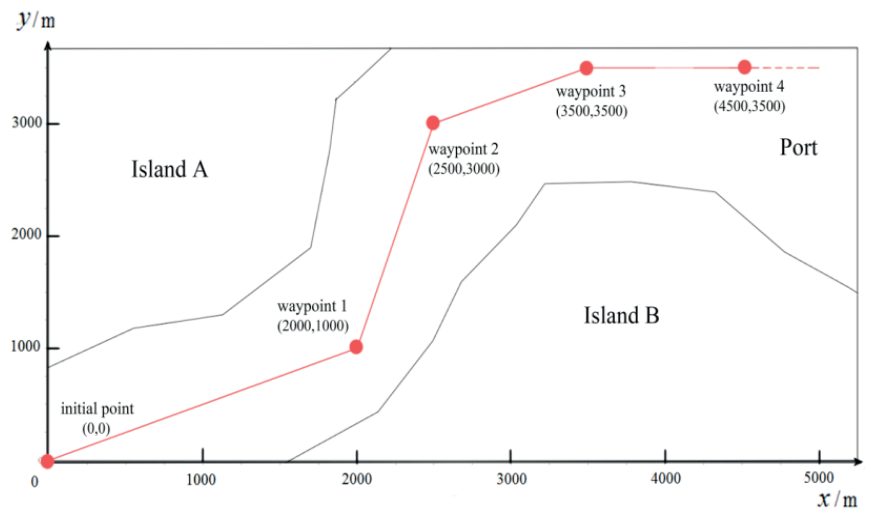

Fig. 5. Ship's route planning

\section{SHIP TRAJECTORY TRACKING SIMULATION AND VALIDATION WITH AUTONOMOUS TRACKING SYSTEM}

To consider the accuracy and stability of the method, we used the fourth-order Runge-Kutta method to solve the ordinary differential equation system. The research object in this paper was the container ship A. The main parameters of hull, propeller and rudder for this ship are shown in Table 2.

Tab.2. Parameters of hull, propeller and rudder for container ship A

\begin{tabular}{|c|c|}
\hline Length between perpendiculars $L / \mathrm{m}$ & 172 \\
\hline Beam B/m & 32.2 \\
\hline Fore draft $d_{f} / \mathrm{m}$ & 11.3 \\
\hline Aft draft $d / \mathrm{m}$ & 11.3 \\
\hline displacement $\nabla / \mathrm{m}^{3}$ & 50500 \\
\hline Wetted area $S / \mathrm{m}^{2}$ & 8201 \\
\hline Block coefficient $C_{b}$ & 0.807 \\
\hline Propeller diameter $D / m$ & 5.9 \\
\hline Pitch diameter ratio $P / D$ & 0.8 \\
\hline Blade number $Z$ & 4 \\
\hline Area ratio $E A R$ & 0.7 \\
\hline Rudder aspect ratio $\lambda_{R}$ & 1.7872 \\
\hline Rudder height $H / \mathrm{m}$ & 8.4 \\
\hline Rudder area $A_{R} / \mathrm{m} 2$ & 39.48 \\
\hline
\end{tabular}

The assumed initial parameters of ship motion were as follow: initial speed $u_{0}=7.2 \mathrm{~m} / \mathrm{s}$, course $\Psi_{0}=26.56$, and initial rotational speed of propeller $n_{0}=3 \mathrm{rps}$. The rotational speed of the propeller remained unchanged until the ship reached waypoint 4. The simulation route is shown in Figure 6.

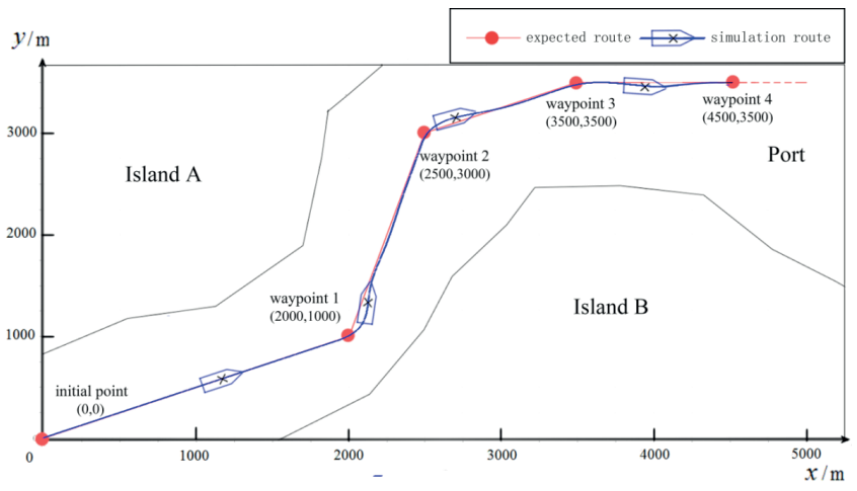

Fig. 6. Comparison of simulation route and expected route

From the simulation result we can see that the ship almost passes through all waypoints of the expected route. A relatively large offset is observed after passing waypoints 1 and 2 by the ship, because the heading changes noticeably in these areas. However, the offset is smaller than the ship length, and the simulation route corresponds to the expected route, so the route stability is maintained.

Time-dependent changes of longitudinal speed $u$, transverse speed $v$, rate of turning $r$, heading $\Psi$, and rudder $\delta$ of the ship, which were recorded in the simulation are shown in Figure 7.

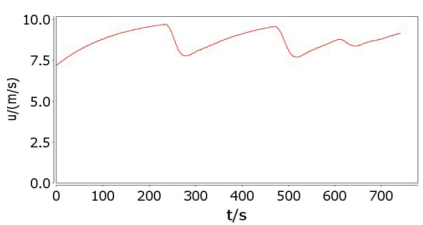

(a)longitudinal speed

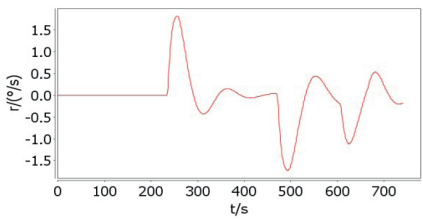

(c)rate of turning

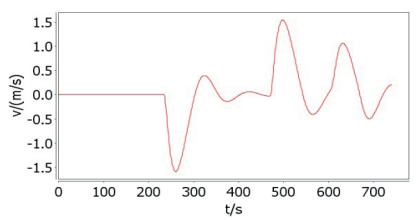

(b)transverse speed

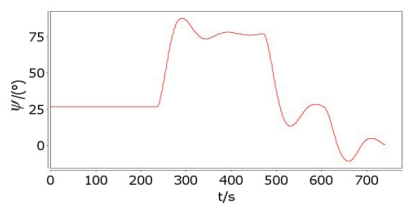

(d)heading

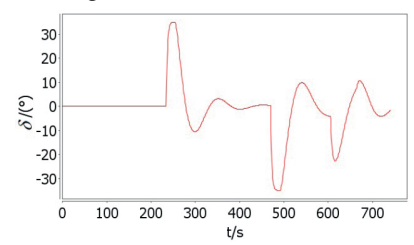

(e)rudder

Fig. 7. Ship's attitude, speed and rudder changes in tracking system simulation

We can see in Figure 7 that all motion parameters change noticeably when the ship approaches each waypoint, especially waypoints 1,2 and 3, because there is large course-alerting at 
each point. The results of the simulation show that the crosstrack error converges smoothly and without overshoot or buffeting, while the course and rudder angle oscillate around a fixed value. The control effect is satisfying and robust.

\section{CONCLUSIONS}

In the paper, mathematical modeling, numerical calculation method, programming language design, and graphic simulation method, are applied synthetically. The automatic navigation and trajectory tracking control system of 3-DOF in the plane for a ship is established. The following conclusions can be formulated based on the results of modeling and simulation.

With respect to ship nonlinear tracking control systems, this article has redesigned the cross-track error $y$ and the course deviation $\varphi$ by taking advantage of strict bounds of the hyperbolic tangent function and the integral term to improve the effect of trajectory tracking. The nonlinear and robust control algorithm proposed in this work and the linear track controller derived using the sliding-mode and incremental feedback methods can effectively overcome the problem of unknown origin. Moreover, the tracking simulation performed for a container ship proves the feasibility of the sliding mode algorithm and the automatic navigation and trajectory tracking system. Good congruency is observed between the simulation route and the expected route. In a word, the simulation of ship trajectory tracking has been successfully conducted and the simulation system has been successfully established.

\section{ACKNOWLEDGEMENT}

This work was supported by "Natural Science Foundation of Liaoning Province, China"(Number:201602084) and "The Fundamental Research Funds for the Central Universities" (Number: 3132016002,3132016006, 3132016008).

\section{REFERENCES}

1. Tao Liu, Zaopeng Dong, Hongwagn Du., et al. (2017).Path following control of the underactuated USV based on the improved line-of-sight guidance algorithm. IEEE Polish Maritime Research. 1 (93),Vol. 24; pp. 3-11.

2. Dong Z.P., Wan L., Liu T., et al. (2016).Horizontal-plane trajectory-tracking control of an unmanned maritime vehicle in the presence of ocean currents. International Journal of Advanced Robotic Systems, 13: 83, 1-14.

3. Larrazabal J.M., Penas M.S.( 2016). Intelligent rudder control of an unmanned surface vessel. Expert Systems with Applications, 55: 106-117.

4. H. Ashrafiuon, K.R. Muske, L.C. Mcninch, (2010).Review of nonlinear tracking and set-point control approaches for autonomous under-actuated marine vehicles, in: American Control Conference, Baltimore, USA, IEEE, pp. 5203-5211.

5. W. Xie, B. Ma, (2015).Robust global uniform asymptotic stabilization of underactuated surface vessels with unknown model parameters, Int. J. Robust Nonlinear Control , 25:1037-1050.

6. K.Y. Pettersen, T.I. Fossen, (2000).Underactuated dynamic positioning of a ship-experimental results, IEEE Trans. Control Syst. Technol, 8 (5) 863-891

7. K.D. Do,(2010). Practical control of underactuated ships, Ocean Eng, 37 (13) 1111-1119

8. J. Ghommam, M. Saad, (2014).Backstepping-based cooperative and adaptive tracking control design for a group of underactuated AUVs in horizontal plan, Int. J. Control, 87 (5) 1076-1093.

9. R. Yu, Q. Zhu, G. Xia, Z. Liu, (2012).Sliding mode tracking control of an underactuated surface vessel, IET Control Theory Appl. 6 (3):461-466

10. A.M. Lekkas, T.I. Fossen, (2014).Trajectory tracking and ocean current estimation for marine underactuated vehicles, IEEE Conference on Control Applications, IEEE, Juan Les Antibes, France, pp. 905-910.

11. M.E. Serrano, G.J.E. Scaglia, S.A. Godoy, et al., (2014). Trajectory tracking of underactuated surface vessels: A linear algebra approach, IEEE Trans. Control Syst. Technol, 22 (3) 1103-1111.

12. E. Lefeber, K.Y. Pettersen, H. Nijmeijer, (2003).Tracking control of an underactuated ship, IEEE Trans. Control Syst. Technol. 11 (1) 52-61.

13. E. Peymani, T.I. Fossen, (2015).Path following of underwater robots using Lagrange multipliers, Robotics Auton. Syst, 67:44-52

14. J. Pan, K.D. Do, (2006).Global robust adaptive path following of underactuated ships, Automatica 42 :1713-1722.

15. Mahmut Reyhanoglu, Arjan van der Schaft, N. Harris Mcclamroch, Kolmanovsky. (1999). Dynamics and Control of a class of underactuated Mechanical Systems. IEEE Transactions on Automatic Control. Vol(44), No.9:1663, 1671.

16. Chwa, D.(2011), Global tracking control of underactuated ships with input and velocity constraints using dynamic surface control method. IEEE Transactions on Control Systems Technology, 19,1357-1370 
17. Huang, J., Wen, C., Wang, W., et. al. (2015), Global stable tracking control of underactuated ships with input saturation. Systems \& Control Letters, 85, 1-7.

18. Li, J.-H.(2016), Path tracking of und ships with general form of dynamics International Journal of Control, 89, 506-517.

19. Shojaei, K.(2015), Neural adaptive robust control of underactuated marine surface vehicles with input saturation. Applied Ocean Research, 53,267-278.

20. GUO Chen, WANG Yang, SUN Fu-chun, et. al. (2009). Survey for motion control of underactuated surface vessels. Control and Decision ,24(03):321-329.

21. Bu Ren-xiang, LIU Zheng-jiang, HU Jiang-qiang.(2007). Straight-path Tracking control of underactuated ships using dynamic nonlinear sliding. J Tsing Univ (Sci\&Tech) ,47(02):1880-1883.

22. P. Encarnacao, A. Pacoal, and M. Arcak. (2000).Path following for autonomous marine craft. Proc. of the 5th IFAC Conf. on MCMC, Aalborg, Denmark, pp.117-122.

23. T. Temel, H. Ashrafiuon. (2015).Sliding-mode speed controller for tracking of underactuated surface vessels with extended Kalman filter .ELECTRONICS LETTERS 19th,51(06) :467-469.

24. Zhang R, Chen Y B, Z Q Sun, et. al. (1998). Path control of a surface ship in restricted waters using sliding model. Proc. of the 37th IEEE CDC, FL,pp.3195-3200.

25. Sahu, B.K., Subudhi, B. (2014). Adaptive tracking control of an autonomous underwater vehicle. Int. J. Autom. Comput. 11(3), 299-307

26. Encarnacao, P.; Pascoal, A. (2001). Combined trajectory tracking and path following: an application to the coordinated control of autonomous marine craft Decision and Control. Proceedings of the 40th IEEE Conference .Vol.1:964-969.

27. Do KD (2015). Global inverse optimal tracking control of underactuated omni-directional intelligent navigators (ODINs).Journal of Marine Science and Application, 14(1), $1-13$.

28. LI Tie-shan. (2005).Nonlinear Design on StraightTrajectory-Keeping Control for ships .Da Lian Maritime University, Da Lian, China.

29. Chen M, Ge SS, How VE, et. al. (2013). Robust adaptive position mooring control for marine vessels. IEEE Transaction on Control Systems Technology;21(2):395-409.
30. Mou Chen, Bing Jiang, Rongxin Cui. (2016).Actuator fault-tolerant control of ocean surface vessels with input saturation[J]. INTERNATIONAL JOURNAL OF ROBUST AND NONLINEAR CONTROL; 26:542-564.

31. Cheng Liu, Zao-jian Zou, Tie-shan Li. (2015). Path following of underactuated surface vessels with fin roll reduction based on neural network and hierarchical sliding mode technique[J]. Neural Comput \& Applic 26:1525-1535.

32. S. Bououden, M. Chadli, H. R. Karimi.(2013).Fuzzy sliding mode controller design using Takagi-Sugeno modelled nonlinear systems. Mathematical Problems in Engineering. Vol. $2013: 1-7$.

33. LI Ronghui, LI Tieshan, BU Renxiang. (2013).Disturbance Decoupling Control Based Trajectory Tracking for Underactuated Ships. Proceedings of the 32nd Chinese Control Conference. July 26-28 ,Xi'an, China:8108-8113.

34. T. Li, R. Li ,J. Li.(2012) Decentralized adaptive neural control of nonlinear systems with unknown time delays, Nonlinear Dynamics, 67(3): 2017-2026.

35. Qian D, Tong S, Yi J (2013) Adaptive control based on incremental hierarchical sliding mode for overhead crane systems. Appl Math Inf Sci 7(4):1359-1364.

36. Q. Yang, Z. Yang, Y. Sun. (2012). Universal neural network control of MIMO uncertain nonlinear systems. IEEE Transactions on Neural Networks. Vol. 23:1162-1169,

37. Cheng Liu, Zaojian Zou, Jianchuan Yin. (2015).Trajectory tracking of underactuated surface vessels based on neural network and hierarchical sliding mode. J Mar Sci Technol ,Vol 20:322-330.

38. Bu, Ren-Xiang; Liu, Zheng-Jiang; Li, Tie-Shan.(2007). Iterative sliding mode based increment feedback control and its application to ship autopilot. Journal of Harbin Engineering University, 28:268-272. 


\section{CONTACT WITH THE AUTHORS}

Yong Liu

e-mail: liuyong_dlmu@163.com

Dalian Maritime University

No.1, Linghai RD

116000 Dalian

\section{China}

Renxiang Bu

e-mail: liushuangou0@163.com

Dalian Maritime University

No.1, Linghai RD

116000 Dalian

China

Xiaori Gao

e-mail:425339930@qq.com

Dalian Maritime University

No.1, Linghai RD

116000 Dalian

China 